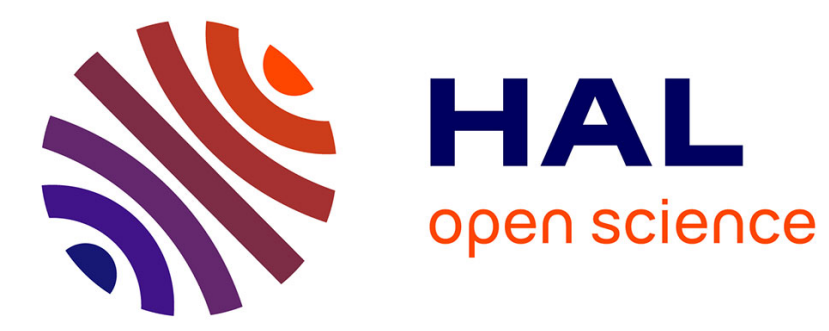

\title{
Early-Drop based Hybrid ARQ in a Cross-layer context
}

Sébastien Marcille, Philippe Ciblat, Christophe Le Martret

\section{To cite this version:}

Sébastien Marcille, Philippe Ciblat, Christophe Le Martret. Early-Drop based Hybrid ARQ in a Cross-layer context. 22nd IEEE Personal Indoor Mobile Radio Communications - PIMRC'11 - Wide Area Cellular Communications, Sep 2011, Canada. pp.1521-1525. hal-00644016

\section{HAL Id: hal-00644016 https://hal.science/hal-00644016}

Submitted on 23 Nov 2011

HAL is a multi-disciplinary open access archive for the deposit and dissemination of scientific research documents, whether they are published or not. The documents may come from teaching and research institutions in France or abroad, or from public or private research centers.
L'archive ouverte pluridisciplinaire HAL, est destinée au dépôt et à la diffusion de documents scientifiques de niveau recherche, publiés ou non, émanant des établissements d'enseignement et de recherche français ou étrangers, des laboratoires publics ou privés. 


\section{Early-Drop based Hybrid ARQ in a Cross-layer context}

\author{
S. Marcille ${ }^{\dagger *}$, P. Ciblat ${ }^{\dagger}$ \\ $\dagger$ COMELEC Dept. \\ Telecom Paristech, Paris, France \\ e-mail: \{sebastien.marcille,philippe.ciblat\} @ telecom-paristech.fr
}

\author{
C. J. Le Martret* \\ * SNE/SPM \\ Thales Communications, Colombes, France \\ e-mail: christophe.le_martret@fr.thalesgroup.com
}

\begin{abstract}
We aim to study a specific cross-layer optimized Hybrid Automatic Retransmission reQuest (HARQ) introduced in [1] and called early-drop HARQ. We remind that in [1], instead of giving a transmission credit per MAC packet (as usually done), it is proposed to share the transmission credit among all the MAC packets belonging to the same IP packet. The early-drop version stops the retransmission as soon as the number of remaining MAC packets is higher than the number of possible transmission attempts. As the early-drop has never been analyzed neither through simulations nor analytical derivations, the purpose of this paper is to fill this gap. As the packet error rate and the delay are not modified, we only focus on the efficiency which is expressed in closed-form.
\end{abstract}

\section{INTRODUCTION}

Hybrid ARQ (HARQ) techniques are promising solutions for high data rates wireless systems, such as LTE [2] or Wimax. Indeed, these techniques enable one to obtain a relevant trade-off between a low packet error rate (via the Forward Error Correction (FEC)), and a good efficiency adapted to the instantaneous channel quality via the repetition mechanism, at the cost of a controllable delay.

The literature on HARQ mechanisms are usually twofold: on the one hand, it is of interest to find powerful error correcting codes (see [3]-[5] for the physical layer, [6] for the application layer); on the other hand, it is of interest to analyze the HARQ mechanisms through closed-form expressions for relevant metrics such as the packet error rate (PER), the delay, and the efficiency (see [7]-[10] which only focus on the MAC layer).

In order to improve the HARQ benefit at IP layer, it is proposed in [1] to operate a cross-layer optimization between the MAC and the IP layers. The idea was the following: let us consider that an IP packet is fragmented into $N$ MAC packets on which HARQ is applied. Usually, each MAC packet has its own transmission credit. Such an approach can be called Fragment Based Strategy (FBS). Instead, in [1], the transmission credit is shared among the $N$ MAC packets belonging to the same IP packet. This approach is called IP Based Strategy (IBS). It has been observed that the PER is improved at the expense of a higher delay. The efficiency, as seen in [11], is in contrast very similar.

In [11]-[13], a unified framework for analyzing analytically any type of HARQ at IP layer with and without cross-layer optimization has been developed. Closed-form expressions for most current HARQ metrics (PER, efficiency and delay) have been provided for FBS and IBS.

A way for improving the IBS efficiency, called early drop, has been roughly mentioned in [1]. The idea is to stop the IP packet transmission as soon as the number of remaining MAC packets is higher than the remaining number of transmission attempts. Intuitively, this enables one to save useless transmissions, when the IP packet is conditioned to be lost. Nevertheless, this new way has been investigated neither through extensive simulations nor analytical derivations. Therefore we propose, in this paper, to express in closed-form the efficiency of the early-drop based HARQ. Notice that the other metrics (PER and delay) are not modified.

The paper is organized as follows: in Section II, we describe more precisely the early-drop HARQ mechanism and introduce some notations. In Section III, we provide the new closed-form expressions for the efficiency in the general case and some particular cases (ARQ, etc). We also prove that the early drop "trick" improves the efficiency for all types of HARQ. In Section IV, numerical illustrations are provided. Finally Section V is devoted to concluding remarks.

\section{EARLY-DROP HARQ MECHANISM}

At the transmitter side, the MAC layer has to transmit several IP packets of length $L_{\mathrm{IP}}$. Each IP packet is split into $N$ MAC packets of length $L_{\mathrm{MAC}}=L_{\mathrm{IP}} / N$. From each MAC packet, some subblocks are generated in order to be transmitted by the PHY layer. Due to the lack of space and for the sake of simplicity, we hereafter only introduce the socalled Incremental Redundancy HARQ (IR-HARQ). Notice that our later derivations actually hold for any type of HARQ.

Each MAC packet for which a header and a CRC have been added is encoded by a FEC code of rate $R_{0}$ (known as the mother code). The encoded MAC packet is next split into $t_{0}$ PHY packets, usually through puncturing of the mother code. The PHY packets (denoted PP) associated with the same MAC packet are then numbered as $\{\mathrm{PP}(i)\}_{i=1}^{t_{0}}$. The transmitter transmits sequentially $\mathrm{PP}(1)$ up to $\mathrm{PP}\left(t_{0}\right)$ upon error detection. If the MAC packet is still not received after the transmission of the last $\mathrm{PHY}$ packet $\mathrm{PP}\left(t_{0}\right)$, the first PHY packet $\mathrm{PP}(1)$ is transmitted again and so on. These PHY packets are sent through a propagation channel (that may be Gaussian, Rayleigh, Frequency-Selective, etc). The length of 
the $i$-th PHY packet is $\delta_{i}$ for $i \in\left\{1, \ldots, t_{0}\right\}$, and we denote by $w_{k}=\sum_{i=1}^{k} \delta_{i} \bmod t_{0}$ the number of channel used to receive a single MAC packet in $k$ transmissions.

At the receiver side, the incoming PHY packet is decoded and sent to the MAC layer which decides whether to send back an ACKnowledgment (ACK) or a Negative ACKnowledgment (NACK) to the transmitter accordingly. To make a decision on the MAC packet, the receiver has the following sequential process: checking the $\mathrm{CRC}$ for $\mathrm{PP}(1)$ after decoding; if $\mathrm{PP}(1)$ is not correctly received, it sends a NACK and it receives afterwards $\mathrm{PP}(2)$. Then checking the CRC after decoding the concatenation of both previous PHY packets (corresponding to a FEC of lower rate), and so on until the reception of $\mathrm{PP}\left(t_{0}\right)$ which is concatenated with the $\left(t_{0}-1\right)$ previous PHY packets and then decoded as the mother code of rate $R_{0}$, followed by the CRC checking. Then, if the MAC packet is not received after $\operatorname{PP}\left(t_{0}\right)$ reception and the transmission credit is not reached, the received packet memory is flushed and the process starts again.

We recall that conventionnally (FBS) the transmission credit is the same for all MAC packets. The IBS approach, introduced in [1], leads to an ARQ scheme enhancement by providing a global transmission credit, denoted by $C$, to the set of MAC packets belonging to the same IP packet. Notice that a straightforward extension to HARQ schemes has been given in [11].

The early-drop (ED) strategy can only be applied to the IBS context. This technique allows a transmitter to discard the IP packet at the $j$-th MAC packet before using its $C$ transmissions, if the remaining transmission credit becomes lower than $N-j$ (the number of remaining MAC packets).

\section{NEW CLOSED-FORM EXPRESSION FOR EFFICIENCY}

\section{A. General case}

As the ED approach only modifies the packet processing when the IP packet will not be correctly received, the PER and the delay (defined as the average number of packet transmissions when an IP packet is successfully received) are identical to those given in [1], [11], [12]. In contrast, the efficiency is modified.

As said in [13], the efficiency of any type of HARQ mechanism at the IP layer can be written

$$
\eta=\frac{L_{\mathrm{IP}}(1-\Pi)}{\Pi \check{n}+(1-\Pi) \hat{n}},
$$

where

- $\Pi$ stands for the IP packet error rate. The closed-form expression for $\Pi$ given in [11], [13] remains valid for the ED approach.

- $\hat{n}$ is the average number of bits sent given that IP packet has correctly been received. The closed-form expression given in [12] for $\hat{n}$ remains valid for ED.

- $\check{n}$ is the average number of bits sent given that the current IP packet reception fails. The closed-form expression given in [11] for $\check{n}$ is modified by the ED technique since ED does not manage the transmission credit in the same way than classic IBS when the IP packet fails. Therefore our main goal is now to find a closed-form expression for $\check{n}$.

We denote with $\check{n}^{\text {ed }}$ the average number of bits sent given that the current IP packet failed when ED is employed. We have to enumerate each MAC packets combination corresponding to a failure of the IP packet. All these combinations describe the event $\mathcal{E}$ that can be decomposed as follows: $\mathcal{E}=\cup_{\ell=1}^{N} D(\ell)$ where

- $D(1)=\{$ MAC packet \#1 consumes $C-N+2$ credits $\}$,

- $D(\ell)=\{$ MAC packet \#1 OK and MAC packet \#2 OK and $\ldots$ and less than $N-\ell$ credits left during MAC packet \# $\ell$ transmission $\}$ for $\ell \in\{2, \ldots, N-1\}$,

- $D(N)=\{$ MAC packet \#1 OK and $\ldots$ and MAC packet $\#(N-1)$ OK and MAC packet \#N KO with the remaining credit\}.

Before going further, we have to introduce the following notations. Let $p_{1}(k)$ be the probability of receiving one MAC packet in exactly $k$ PHY packet transmissions. Let $q(k)$ be the probability of receiving a MAC packet with errors after $k$ PHY packet transmissions. Let us now explicit the probability of each event $D(\ell)$.

- $\ell=1$ : whenever it is received or not, the MAC packet \#1 consumes at least $C-N+2$ trials which leads to

$$
\operatorname{Pr}\{D(1)\}=q(C-N+1)
$$

and the number of bits sent for this event is equal to $d(1)=w_{C-N+2}$.

- $\ell \in\{2, \ldots, N-1\}$ : let us assume that the MAC packet \#k (with $k \leq \ell-1$ ) has been successfully received and has used $i_{k}$ transmissions. Then, the consumed transmission credit is equal to $m_{\underline{i}}(k)=\sum_{j=1}^{k} i_{j}$ for $k \in\{1, \ldots, \ell-1\}$. The IP packet will not be received if the MAC packet \# $\ell$ consumes at least $C-m_{\underline{i}}(\ell-1)-$ $(N-\ell)+1$ credits, whenever it is received or not. Such an event is denoted by $D_{\underline{i}}(\ell)$. Therefore, we have

$$
\operatorname{Pr}\{D(\ell)\}=\sum_{\underline{i} \in \mathcal{T}_{\ell}} \operatorname{Pr}\left\{D_{\underline{i}}(\ell)\right\}
$$

where $\mathcal{T}_{\ell}=\left\{\underline{i} \in \mathbb{N}_{*}^{\ell-1} \mid m_{\underline{i}}(\ell-1)=\sum_{k=1}^{\ell-1} i_{k}<C-\right.$ $N+\ell\}$ and

$$
\operatorname{Pr}\left\{D_{\underline{i}}(\ell)\right\}=q\left(C-m_{\underline{i}}(\ell-1)-N+\ell\right) \prod_{k=1}^{\ell-1} p_{1}\left(i_{k}\right)
$$

and the number of bits sent for the event $D_{\underline{i}}(\ell)$ is equal to $d_{\underline{i}}(\ell)=r_{\underline{i}}(\ell-1)+w_{C-m_{\underline{i}}}(\ell-1)-(N-\ell)+1$ with $r_{\underline{i}}(\ell)=$ $\sum_{k=1}^{\ell^{-}} w_{i_{k}}$.

- $\underline{\ell=N}$ : similar derivations lead to

$$
\operatorname{Pr}\{D(N)\}=\sum_{\underline{i} \in \mathcal{T}_{N}} \operatorname{Pr}\left\{D_{\underline{i}}(N)\right\}
$$

where $D_{\underline{i}}(N)$ is defined as in Eq. (3) by putting $\ell=N$. However, the number of transmitted bits for the event $D_{\underline{i}}(N)$ is $d_{\underline{i}}(N)=r_{\underline{i}}(N-1)+w_{C-m_{\underline{i}}(N-1)}$. 
The delay $\check{n}^{e d}$ corresponds to the sum of the number of bits $d_{\underline{i}}(\ell)$ weighted by the probability of the event $D_{\underline{i}}(\ell)$ divided by the IP packet error rate. Therefore, we finally have

$$
\check{n}^{e d}=\frac{1}{\Pi}\left(d(1) \operatorname{Pr}\{D(1)\}+\sum_{\ell=2}^{N} \sum_{\underline{i} \in \mathcal{T}_{\ell}} d_{\underline{i}}(\ell) \operatorname{Pr}\left\{D_{\underline{i}}(\ell)\right\}\right) .
$$

The term $1 / \Pi$ occurs since $\check{n}$ has been calculated conditionnally to the fact that the IP packet is not correctly received.

We are now able to obtain the following result. The proof is reported in Appendix A.

Result 1. Let $\eta$ be the efficiency of an IBS based HARQ at the IP level. Let $\eta^{\text {ed }}$ be the efficiency of the same system but when early drop is carried out. We have then

$$
\eta^{e d} \geq \eta
$$

\section{B. Particular cases}

a) Equal Packet Length: We now assume that all PHY packets have the same length. This assumption is often done in the literature. For example, when IR-HARQ is considered, if the mother code has a rate $R_{0}=1 / t_{0}$ and the punctured code rates are $\{1 / t\}_{t=1, \ldots, t_{0}}$, then the equal PHY packet length assumption is satisfied.

In that case, when $\sum_{k=1}^{\ell-1} i_{k}=s$ we have

$$
w_{i_{k}}=i_{k} L_{\mathrm{MAC}}, m_{\underline{i}}(\ell-1)=s, r_{\underline{i}}(\ell-1)=s L_{\mathrm{MAC}}
$$

since $\delta_{k}=L_{\mathrm{MAC}}, \forall k$.

Like in the case of different packet lengths, we have to distinguish the three cases:

- $\underline{\ell=1}$ :

$$
d(1) \operatorname{Pr}\{D(1)\}=L_{\mathrm{MAC}}(C-N+2) \operatorname{Pr}\{D(1)\} .
$$

- $\ell \in\{2, \cdots, N-1\}$ : one can easily check that

$$
\mathcal{T}_{\ell}=\bigcup_{s=\ell-1}^{C-N+\ell-1} Q_{s, \ell}
$$

where $Q_{s, \ell}$ is the subset of $\mathcal{T}_{\ell}$ such that $\sum_{k=1}^{\ell-1} i_{k}=s$. As a consequence,

$$
\sum_{\underline{i} \in \mathcal{T}_{\ell}} d_{\underline{i}}(\ell) \operatorname{Pr}\left\{D_{\underline{i}}(\ell)\right\}=\sum_{s=\ell-1}^{C-N+\ell-1} \sum_{\underline{i} \in Q_{s, \ell}} d_{\underline{i}}(\ell) \operatorname{Pr}\left\{D_{\underline{i}}(\ell)\right\} .
$$

When $\underline{i} \in Q_{s, \ell}$, we have

$$
d_{\underline{i}}(\ell)=(C-N+\ell+1) L_{\mathrm{MAC}} .
$$

Therefore

$$
\sum_{\underline{i} \in \mathcal{T}_{\ell}} d_{\underline{i}}(\ell) \operatorname{Pr}\left\{D_{\underline{i}}(\ell)\right\}=L_{\mathrm{MAC}}(C-N+\ell+1) \operatorname{Pr}\{D(\ell)\} .
$$

- $\underline{\ell=N}$ : similar derivations can be done. We have thus

$$
\sum_{\underline{i} \in \mathcal{T}_{N}} d_{\underline{i}}(N) \operatorname{Pr}\left\{D_{\underline{i}}(N)\right\}=L_{\mathrm{MAC}} C \operatorname{Pr}\{D(N)\} .
$$

Finally, we obtain

$$
\begin{aligned}
\check{n}^{e d} & =L_{\mathrm{MAC}}(C-N+1) \\
& +\frac{L_{\mathrm{MAC}}}{\Pi}\left[\sum_{\ell=1}^{N} \ell \operatorname{Pr}\{D(\ell)\}-\operatorname{Pr}\{D(N)\}\right] .
\end{aligned}
$$

b) Type-I HARQ: for this type of HARQ, all the PHY packets are identical, corresponding to the MAC packet eventually encoded by a fixed rate FEC code. Moreover the PHY packets are treated one by one at the receiver side. Therefore, we can work with the expression of $\check{n}^{\text {ed }}$ given in Eq. (6). Due to the simple relation between the PHY packet and the MAC packet, we will be able to exhibit simple closed-form expressions for the terms $\operatorname{Pr}\{D(\ell)\}$. Notice that the ARQ scheme is actually a Type-I HARQ, for which the MAC packets correspond to data without FEC. As the PHY packets are identical and handled independently in the Type-I HARQ context, their error probability is the same and denoted by $\pi_{0}$. Before going further, we have to remind [14] that

$$
\Pi=I_{\pi_{0}}(C-N+1, N)
$$

where $I_{x}(a, b):=B_{x}(a, b) / B(a, b)$ is the regularized Beta function, $B_{x}(a, b)$ is the incomplete Beta function as defined in [15, Eq. (8.391)] and $B(a, b)=B_{1}(a, b)$ the Beta function. From now, we need to evaluate $\operatorname{Pr}\{D(\ell)\}$. One can prove that

$$
\operatorname{Pr}\{D(\ell)\}=\left|\mathcal{T}_{\ell}\right|\left(1-\pi_{0}\right)^{\ell-1} \pi_{0}{ }^{C-N+1}
$$

where $\left|\mathcal{T}_{\ell}\right|$ is the cardinality of the set $\mathcal{T}_{\ell}$. By convention, we put $\left|\mathcal{T}_{1}\right|=1$. One can check that

$$
\left|\mathcal{T}_{\ell}\right|=\sum_{s=\ell-1}^{C-N+\ell-1}\left(\begin{array}{l}
s-1 \\
\ell-2
\end{array}\right)=\left(\begin{array}{c}
C-N+\ell-1 \\
\ell-1
\end{array}\right) .
$$

Therefore it remains to calculate

$$
\sum_{\ell=1}^{N} \ell \operatorname{Pr}\{D(\ell)\}=\sum_{\ell=1}^{N} \ell\left(\begin{array}{c}
C-N+\ell-1 \\
\ell-1
\end{array}\right)\left(1-\pi_{0}\right)^{\ell-1} \pi_{0}^{C-N+1} .
$$

In Appendix B, it is proven that

$$
\begin{aligned}
\sum_{\ell=1}^{N} \ell \operatorname{Pr}\{D(\ell)\} & =\frac{\pi_{0}+K\left(1-\pi_{0}\right)}{\pi_{0}} I_{\pi_{0}}(K, N) \\
& -\frac{\pi_{0}{ }^{K-1}\left(1-\pi_{0}\right)^{N}}{B(K, N)} .
\end{aligned}
$$

with $K=C-N+1$. Finally we have

$$
\begin{aligned}
\check{n}^{e d} & =\left(\frac{\pi_{0}+K}{\pi_{0}}-\frac{\pi_{0}{ }^{K-1}\left(1-\pi_{0}\right)^{N}}{B_{\pi_{0}}(K, N)}\right. \\
& \left.-\left(\begin{array}{c}
C-1 \\
N-1
\end{array}\right)\left(1-\pi_{0}\right)^{N-1} \pi_{0}^{K}\right) L_{\mathrm{MAC} .}
\end{aligned}
$$

We remind that, in the non early-drop case, we had [11]

$$
\check{n}^{\text {ned }}=C L_{\mathrm{MAC}} \text {. }
$$

Clearly, the early-drop analysis is much more complex than the non-early drop case. Nevertheless we succeed to express $\check{n}$ in closed-form through Eq. (7). 


\section{NUMERICAL RESULTS}

In this section, the relevance of early-drop is investigated for different HARQ types. Before going further, we would like to validate our analytical expressions. In Figure 1, we compute expression (5) and we also evaluate the efficiency through extensive Monte-Carlo simulations. For several types of HARQ (ARQ or HARQ with Chase Combining) and channels (Gaussian or Rayleigh), we observe a nice agreement between our expressions and the estimated points.

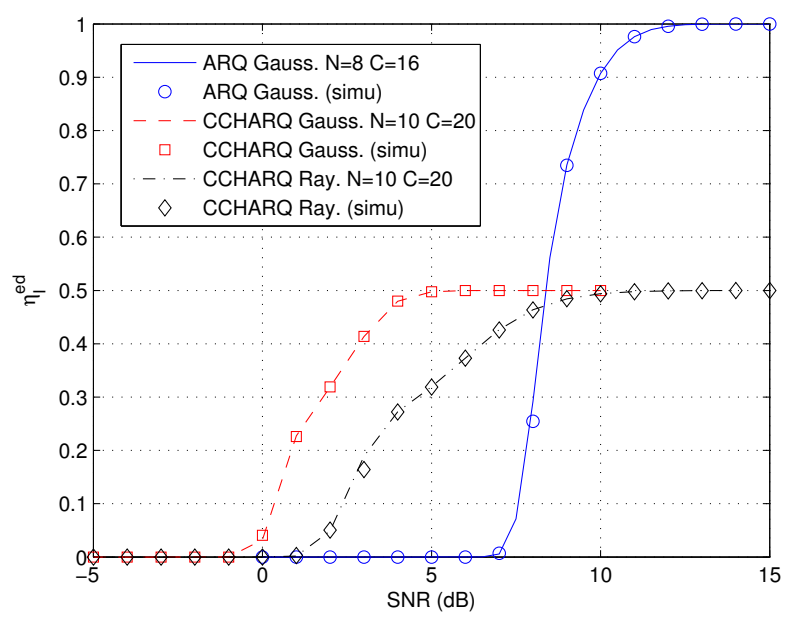

Figure 1. Theoretical and empirical efficiencies versus SNR for different types of HARQ when ED is applied.

For the sake of clarity we will now consider, for instance, an ARQ scheme with QPSK over an AWGN channel. Numerous other simulations have been run for different configurations ; all of them lead to similar comments. Figure 2 illustrates Result 1 for the best configuration we have found, but one can notice that the gain in efficiency is small. Thus, early-drop does not provide a significant gain but only an incremental one.

Let us explain this phenomenon. In Figure 3, we plot (versus SNR) the two terms involved in the denominator of Eq. (1), when early-drop is employed or not. We observe that the highest difference between the terms $\Pi \check{n}$ occurs at low and medium SNR. As soon as the SNR becomes large enough, ED does not provide improvement since $\Pi \check{n}$ (circles) and $\Pi \check{n}^{\text {ed }}$ (squares) have quite the same value. Hence, at low SNR the gain brought by $\mathrm{ED}$ (in $\Pi \check{n}$ ) is potentially important. But the corresponding efficiency is very low (near 0 ) for both approaches, and the improvement keeps very small and thus useless in absolute. However, at medium SNR the efficiency is slightly improved.

In Table I we report, for different channels and HARQ mechanisms, the relative gains in efficiency defined as

$$
G=\frac{\eta^{e d}-\eta}{\eta}
$$

and averaged over uniform SNR intervall.

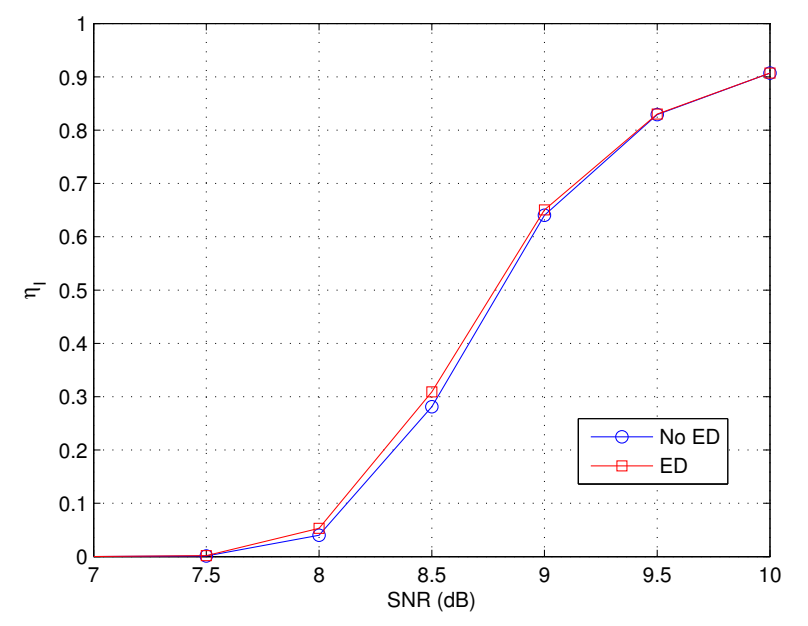

Figure 2. Efficiency versus SNR (ARQ scheme, AWGN channel, QPSK constellation, $N=14$, and $C=21$ ).

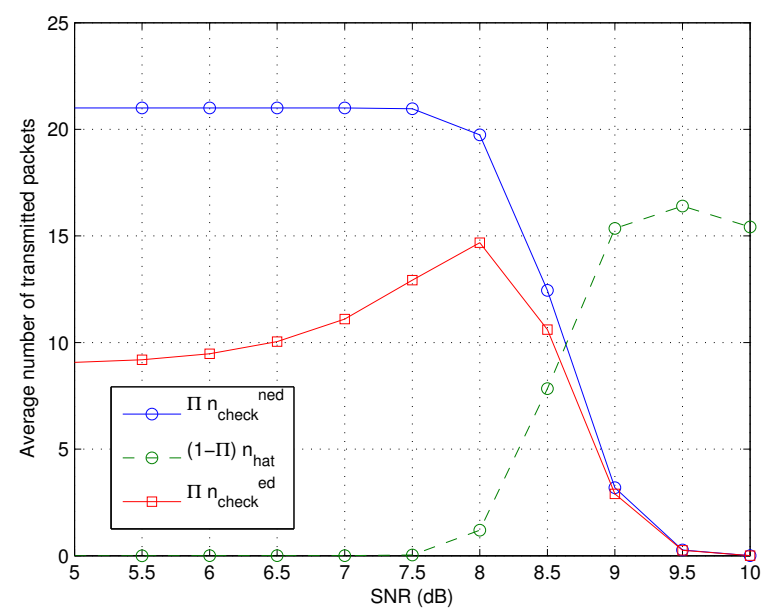

Figure 3. $\Pi \check{n}$ and $(1-\Pi) \hat{n}$ versus SNR (ARQ scheme, AWGN channel, QPSK constellation, $N=14$, and $C=21$ ).

\begin{tabular}{|c|c|c|}
\hline & $G(\%)$ over AWGN & $G(\%)$ over Rayleigh \\
\hline ARQ & 12.4 & 17.1 \\
\hline CC-HARQ & 4.0 & 3.8 \\
\hline IR-HARQ & 2.6 & 1.8 \\
\hline
\end{tabular}

Table I

AVERAGE RELATIVE EFFICIENCY GAIN FOR DIFFERENT HARQ TYPES $(N=8, C=16)$.

\section{CONCLUSION}

We have deeply analyzed the so-called early-drop technique, well adapted to cross-layer designed HARQ schemes. A closed-form expression for the efficiency has been derived, and it has been shown that ED can improve the efficiency without degrading the other performance metrics. However, the relative gain is quite small (from $1 \%$ to $17 \%$ ), but remains 
of interest considering the free cost implementation of ED in an IBS framework.

\section{APPENDIX}

\section{A. Proof of Result 1}

In non-early drop context, a more precise description than $D_{\underline{i}}(\ell)$ is needed since we have to know how the MAC packets $\# \ell^{\prime}$ (with $\ell^{\prime}>\ell$ ) are handled. Therefore we can decompose $D_{\underline{i}}(\ell)$ as follows: $D_{\underline{i}}(\ell)=\cup_{\underline{i}^{\prime}} D_{\underline{i}, \underline{\prime}^{\prime}}(\ell)$ where $D_{\underline{i}, \underline{i}^{\prime}}(\ell)$ represents a certain way of handling the remaining $N-\ell-1$ MAC packets given that the $\ell$ first MAC packets are handled as in $D_{i}(\ell)$. Then, we have to replace in Eq. (5)

$$
\sum_{\underline{i} \in \mathcal{T}_{\ell}} d_{\underline{i}}(\ell) \operatorname{Pr}\left\{D_{\underline{i}}(\ell)\right\}
$$

with

$$
\sum_{\underline{i} \in \mathcal{T}_{\ell}} \sum_{\underline{i}^{\prime}} d_{\underline{i, i^{\prime}}}(\ell) \operatorname{Pr}\left\{D_{\underline{i}, \underline{i}^{\prime}}(\ell)\right\}
$$

where $d_{\underline{i}, \underline{i}^{\prime}}(\ell)$ is the cost in packets of the event $D_{\underline{i}, \underline{i}^{\prime}}(\ell)$. As in early drop context the transmission stops as soon as $D_{\underline{i}}(\ell)$ occurs, we have

$$
d_{\underline{i}, \underline{i}^{\prime}}(\ell) \geq d_{\underline{i}}^{e d}(\ell)
$$

which implies that $\check{n} \geq \check{n}^{\text {ed }}$ and concludes the proof.

\section{B. Simplification for Type-I HARQ}

The purpose is to find $\sum_{\ell=1}^{N} \ell \operatorname{Pr}\{D(\ell)\}=\pi_{0}{ }^{C-N+1} f(1-$ $\left.\pi_{0}\right)$, where $f$ is the (analytical) function defined for $x \in[0,1]$ by

$$
f(x)=\sum_{\ell=1}^{N} \ell\left(\begin{array}{c}
C-N+\ell-1 \\
\ell-1
\end{array}\right) x^{\ell-1} .
$$

Our approach will be to find a closed-form expression for a primitive $F$ of $f$,

$$
F(x)=\sum_{\ell=1}^{N}\left(\begin{array}{c}
C-N+\ell-1 \\
\ell-1
\end{array}\right) x^{\ell}
$$

and then to calculate its derivative. Due to the page limitation, we only give a sketch of the proof, and some simplifications are left to the reader. First, by using the symmetry property $\left(\begin{array}{l}n \\ k\end{array}\right)=\left(\begin{array}{c}n \\ n-k\end{array}\right), \forall n \geq k$ and an index reorganization, we obtain that

$$
F(x)=\frac{G(x)}{x^{C-N}} \text { with } G(x)=\sum_{\ell=C-N+1}^{C}\left(\begin{array}{c}
\ell-1 \\
C-N
\end{array}\right) x^{\ell} .
$$

Then, by using the Pascal identity for binomial coefficients [15, Eq. (3.1.4], it can be shown that $G$ satisfies the following differential equation

$$
y-\frac{x(1-x)}{K} y^{\prime}=\mu x^{C+1}
$$

with $\mu=\left(\begin{array}{l}C \\ K\end{array}\right)$ and $K=C-N+1$. Standard resolution methods can be used to solve Eq. (9), and we have

$$
G(x)=\left(\frac{x}{1-x}\right)^{K}\left(\lambda-K \mu B_{x}(N, K)\right) .
$$

Due to [15, Eq. (6.2.2)], we have $\mu K=1 / B(N, K)$. Thus

$$
F(x)=\frac{x}{(1-x)^{K}}\left(\lambda-I_{x}(N, K)\right) .
$$

In order to characterize $\lambda$, let us consider $\Pi$. One can check that

$\Pi=\sum_{\ell=1}^{N} \operatorname{Pr}\{D(\ell)\}=\frac{\pi_{0}{ }^{K}}{1-\pi_{0}} F\left(1-\pi_{0}\right)=\lambda-I_{1-\pi_{0}}(N, K)$.

As $\lim _{\pi_{0} \rightarrow 1} \Pi=1$ and $\lim _{\pi_{0} \rightarrow 1} I_{1-\pi_{0}}(N, K)=0$, we have $\lambda=1$. Thus

$$
F(x)=\frac{x}{(1-x)^{K}}\left(1-I_{x}(N, K)\right) .
$$

Moreover, as $1-I_{x}(b, a)=I_{1-x}(a, b)$, we finally have

$$
F(x)=\frac{x}{(1-x)^{K}} I_{1-x}(K, N) .
$$

To find the final result, just take the derivative of $F($.$) given$ in Eq. (10).

\section{REFERENCES}

[1] Y. Choi, S. Choi, and S. Yoon, "MSDU-based ARQ scheme for IP-level performance maximization," in Global Telecommunications Conference (GLOBECOM), vol. 5. IEEE, Oct. 2005, pp. 2495-2499.

[2] S. Sesia, I. Toufik, and M. Baker, LTE: the Long Term Evolution - From theory to practice. Wiley, 2009.

[3] J. Hagenauer, "Rate-compatible punctured convolutional codes (RCPC codes) and their applications," IEEE Trans. Commun., vol. 36, no. 4, pp. 389-400, Apr. 1988.

[4] S. Sesia, G. Caire, and G. Vivier, "Incremental redundancy Hybrid ARQ schemes based on Low-Density Parity-Check codes," IEEE Trans. Commun., vol. 52, no. 8, pp. 1311-1321, Aug. 2004.

[5] M. Levorato and M. Zorzi, "Performance analysis of type II Hybrid ARQ with Low-Density Parity-Check codes," in International Symposium on Communications, Control and Signal Processing (ISCCSP), 2008, pp. 804-809.

[6] I. Andriyanova and E. Soljanin, "IR-HARQ schemes with finite-length punctured LDPC codes over the BEC," in International Workshop on Information Theory (ITW). IEEE, 2009.

[7] S. Kallel, "Analysis of a type II Hybrid ARQ scheme with code combining," IEEE Trans. Commun., vol. 38, no. 8, pp. 1133-1137, Aug. 1990.

[8] G. Caire and D. Tuninetti, "The throughput of Hybrid-ARQ protocols for the Gaussian collision channel," IEEE Trans. Inf. Theory, vol. 47, no. 5, pp. 1971-1988, Jul. 2001.

[9] E. Soljanin, R. Liu, and P. Spasojevic̀, "Hybrid ARQ with random transmission assignments," in Advances in network information theory: DIMACS Workshop, Network Information Theory, March 17-19, 2003, Piscataway, New Jersey. AMS Bookstore, 2004, p. 321.

[10] Q. Chen and P. Fan, "Performance analysis of Hybrid ARQ with code combining over interleaved Rayleigh fading channel," IEEE Trans. Veh. Technol., vol. 54, no. 3, pp. 1207-1214, May 2005.

[11] A. Le Duc, C. J. Le Martret, and P. Ciblat, "Packet error rate and efficiency closed-form expressions for cross-layer hybrid ARQ schemes," in Signal Processing and Wireless Communications (SPAWC). IEEE, 2009.

[12] — , "Delay and jitter closed-form expressions for cross-layer hybrid ARQ schemes," in Vehicular Technology Conference (VTC). IEEE, 2009.

[13] — "Efficiency closed-form expressions for any IR-HARQ scheme at the IP level," in Signal Processing and Wireless Communications (SPAWC). IEEE, 2010.

[14] A. Le Duc, C. J. Le Martret, P. Ciblat, and S. Marcille, "Analytical performance derivation of Hybrid ARQ schemes at IP layer," IEEE Trans. Commun., Jan. 2010, submitted.

[15] M. Abramowitz and I. Stegun, Handbook of mathematical functions, with formulas, graphs, and mathematical tables, 20th ed., ser. Applied Mathematics Series. National Bureau of Standards, Jun. 1972, vol. 55. 